Ankit Tyagi ${ }^{1}$, Isaac Boxx, Stephen Peluso ${ }^{1}$, Jacqueline O'Connor ${ }^{1}$, Pocket

Formation and Behavior in Turbulent Premixed Flames, Combust. Flame 211 (2020) 312 - 324.

${ }^{1}$ Pennsylvania State University

The original publication is available at www.elsevier.com

https://doi.org/10.1016/j.combustflame.2019.09.033

(C) <2020>. This manuscript version is made available under the CC-BY-NC-ND 4.0 license http://creativecommons.org/licenses/by-nc-nd/4.0/ 


\title{
Pocket Formation and Behavior in Turbulent Premixed Flames
}

\author{
Ankit Tyagi ${ }^{1}$, Isaac Boxx ${ }^{2}$, Stephen Peluso ${ }^{1}$, Jacqueline O'Connor ${ }^{1}$ \\ ${ }^{I}$ Mechanical Engineering, Pennsylvania State University, University Park, PA, USA \\ ${ }^{2}$ German Aerospace Center (DLR), Stuttgart, Germany
}

\begin{abstract}
Pocket formation is an important characteristic of turbulent premixed flames and understanding pocket behavior is key to developing high-fidelity numerical combustion models. In this study, a dual-burner experiment is used to study pockets in single- and dual-flame configurations and synchronized high-speed OH-planar laser-induced fluorescence and stereoscopic-particle image velocimetry imaging techniques are implemented to track flame pockets and the surrounding flow field. Statistical analysis of pocket origin and fate is performed using a novel tracking algorithm incorporating non-rigid image registration. Results show that pocket formation rates increase as a function of increasing inlet turbulence level; reactant pocket formation increases as a function of downstream distance, whereas product pocket formation decreases. Tracking reactant pocket lifetime shows that a majority of these pockets burn out and displacement speeds are characterized. Product pockets usually merge with the main flame surface, which could have an impact on local flame structure and propagation. Results presented in this study show that pocket behavior in turbulent flames can change local flame dynamics and it is important to capture these effects in sub-grid scale combustion models to accurately predict flame behavior.
\end{abstract}




\section{Nomenclature}

DNS Direct numerical simulation

FOV Field-of-view

FSD Flame surface density

LES Large-eddy simulation

PLIF Planar laser-induced fluorescence

s-PIV Stereoscopic-particle image velocimetry

$A_{\bar{c}} \quad$ Time-averaged flame area based on $\bar{c}$

$H \quad$ Time-averaged flame height

$L_{11} \quad$ Integral length-scale

$P_{1} \quad$ Perimeter of a reactant pocket contour at $t_{0}$

$R e_{w} \quad$ Width-based bulk flow Reynolds number

$R e_{h} \quad$ Hydraulic diameter-based Reynolds number

$R e_{L_{11}} \quad$ Turbulent Reynolds number

$S_{D} \quad$ Reactant pocket displacement speed

$S_{M, C}$

$S_{T}$

Reactant gas consumption due to merging

$S_{T, G C_{\bar{c}}} \quad$ Turbulent global consumption speed based on $\bar{C}$

$U \quad$ Bulk flow velocity

$U_{Z} \quad$ Averaged out-of-plane component velocity inside product pocket

$\bar{c} \quad$ Time-averaged progress variable

$l_{f} \quad$ Laminar flame thickness

$\dot{m}_{R} \quad$ Mass flow rate of reactant gases

$s_{L} \quad$ Unstretched laminar flame speed

$\vec{u}_{\text {pocket }} \quad$ Reactant pocket flame surface velocity

$\vec{u}_{\text {conv }} \quad$ Local convection velocity near pocket flame surface

$u^{\prime} \quad$ Turbulence velocity-scale

$u_{x}^{\prime} \quad x$-component of turbulence velocity scale

$u_{y}^{\prime} \quad y$-component of turbulence velocity scale

$u_{z}^{\prime} \quad z$-component of turbulence velocity scale

$w \quad$ Burner width

$x \quad$ Stream-wise direction

$y \quad$ Cross-stream direction

$Z \quad$ Span-wise direction

$\Delta A \quad$ Two-dimensional area change for a reactant pocket in $\Delta t$

$\lambda_{\text {Taylor }} \quad$ Taylor length-scale

$\rho_{R} \quad$ Mixture density of reactant gases

$\mathcal{R}_{R, p} \quad$ Reactant pocket formation rate

$\mathcal{R}_{P, p} \quad$ Product pocket formation rate 


\section{Introduction}

The formation of flame pockets in turbulent flames is a natural result of turbulence-flame interactions and the resultant flame wrinkling. Flame pockets, for the purposes of this study, are defined as regions of either reactants or products that have separated from the main flame structure. Reactant pockets, or separated regions of reactant gases surrounded by a flame, are typically formed after flame-flame interaction events where the flame is so locally contorted that a flame tunnel closure results in a "pinching" of the flame and the formation of a pocket. Product pockets can be formed as a result of either product-side flame interactions or flame holes and are defined as separated regions of product gases that are found on the reactant side of the flame. Both experiments [1-3] and simulations [4-6] have shown that reactant-side interactions occur more frequently than product-side interactions, which would suggest that reactant pockets are formed more frequently than product pockets.

Most studies of pocket formation used simulation to understand the formation of pockets and the effect that they have on turbulent flame behavior. Work by Denet [7] used two-dimensional direct numerical simulation (DNS) to quantify the frequency of pocket formation; the results show that the number of pockets formed increased as the range of turbulent scales also increased. Further 2D DNS studies by Chen et al. [8] explored the details of the flame interaction processes that lead to reactant pocket formation. Much emphasis was placed on understanding the interaction of different "layers" (preheat layer, reaction layer, etc.) during the interaction. Three primary processes have been observed in the lifetime of reactant pockets. First, a flame channel closes, resulting in a cusp on the main flame and a pocket. Second, cusp recovery occurs on the main flame after the interaction. Third, the flame surrounding the separated pocket continues to propagate, leading to pocket burn out. During tunnel closing and pocket burnout events, thermo- 
diffusive and chemical interactions result in the acceleration of the flames prior to annihilation. However, the time scales associated with the final stage of mutual annihilation and the initial stage of cusp recovery are significantly smaller than diffusive and convective time scales, meaning that these interaction effects alter flame propagation relatively little. Relatively few simulations have considered the behavior of reactant pockets during burnout. Sun and Law [9] used a 1D PREMIX [10] simulation in spherical coordinates to calculate the flame speed as a function of pocket radius. Their findings show that Lewis number plays an important role in controlling both burnout rate and burnout completion, as Lewis number controls whether the high curvature levels at the end of burnout lead to flame extinction or burning enhancement.

A number of experimental studies have measured pocket burnout rates in mostly laminar pockets. Studies by Ibarreta and Driscoll [11] and Baillot and co-workers [12, 13] investigated laminar inwardly propagating flames, essentially reactant pockets, to understand the correlation between burnout rates and the mean flame curvatures for these flames. Their studies showed that mean curvature plays an important role in enhancing the burning rates of these laminar flames. Some recent studies have analyzed burnout rates for reactant pockets originating in the flame tip region of turbulent Bunsen flames. Johchi et al. [14] performed simultaneous velocity and $\mathrm{CH} / \mathrm{OH}$ field measurements for reactant flame pockets and their results showed that the most probable consumption rates were much higher than the unstretched laminar flame speed. Another study by Han et al. [15] studied the effect of $\mathrm{CO}_{2}$ addition on consumption rates of unburned reactant pockets. Their results showed that addition of $\mathrm{CO}_{2}$ does not impact the most probable consumption rates of unburned fine scale pockets and weak correlation exists between pocket size and consumption rates. More recent work conducted by Kim et al. [16] showed a correlation between the number of unburned reactant pockets and integral length-scale with downstream distance. 
Very little treatment of product pockets can be found in the literature. Statistics of product pocket formation were reported by Dunstan et al. [4] and Griffiths et al. [6], indicating that product pocket formation rates increased with turbulence intensity, a result of the high levels of strain required to drive product-side flame interactions. This trend was confirmed experimentally in our recent work in a turbulent Bunsen flame [1]. Recent work by Xu et al. [17] considered the impact that product pockets have on ignition tendencies and flame propagation using three-dimensional DNS and explosive mode analysis. In this work, they showed that the existence of product pockets in the reactant stream acts as a sort of exhaust gas recirculation (EGR), causing preheating and chemical activation of the reactants ahead of the flame and enhancing the explosiveness of that mixture.

While this previous work on flame pockets provides a useful foundation for understanding pocket formation, it has two gaps that we address in the present work. First, we provide statistics of both reactant and product formation rates in highly turbulent Bunsen flames over a range of operating conditions, adding to the little data available. We show that pocket formation happens quite frequently at high turbulence intensities and quantify the spatial dependence of both pocket formation rate and size for a range of turbulence levels. Second, and more importantly, we have not only identified the modes by which pockets are formed, but also tracked them throughout their lifetime. Pockets can not only burn out, but can also re-merge with the flame, which can have a local impact of flame dynamics for both reactant and product pocket mergers. Further, we have quantified reactant pocket burnout rates using pocket displacement speed. Finally, we have considered the different effects that product pockets could have on flame propagation, following the work of Xu et al. [17]. 


\section{Experimental Configuration}

\subsection{Burner Configuration}

The dual burner configuration consists of two identical burners mirrored about the experiment centerline (Figure 1). The exit plane cross-section for each burner is $10 \mathrm{~mm}$ x $100 \mathrm{~mm}$; each burner contains the inlet for the premixed reactants (natural gas and air), two ceramic honeycomb flowstraighteners, and two perforated-plate turbulence generators. The turbulence generation plates have a staggered hole pattern with $3.2 \mathrm{~mm}$ hole-diameters and a $40 \%$ open area. These plates are mounted 30 and $10 \mathrm{~mm}$ upstream of the burner exit plane, resulting in an $18 \%$ turbulence intensity averaged along the burner width, normalized by the bulk flow velocity. This turbulence intensity is based on all three components of velocity. Two types of pilot flames (anchoring and backsupport) are utilized in each burner to provide adiabatic combustion products around the flames. A translation stage is used to change fields-of-view (FOVs) for high-speed laser measurements; data from each field of view are not obtained concurrently but instead sequentially on the same day. Changes from dual-flame to single-flame configuration can be made in this experiment by attaching the back-support pilot flame of one burner as a secondary pilot to the other burner. More details on the burner dimensions can be found in our previous work $[1,18,19]$. 


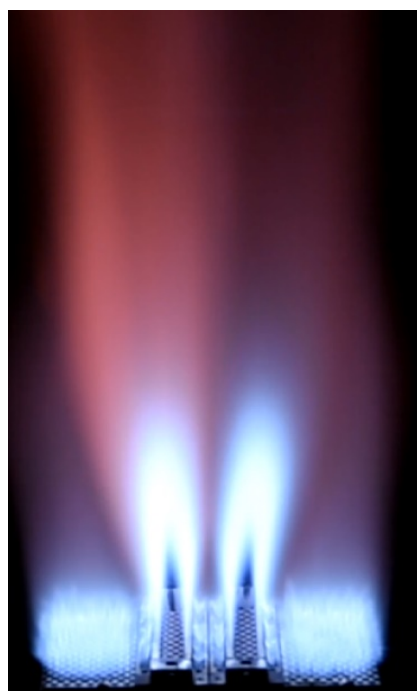

(a)

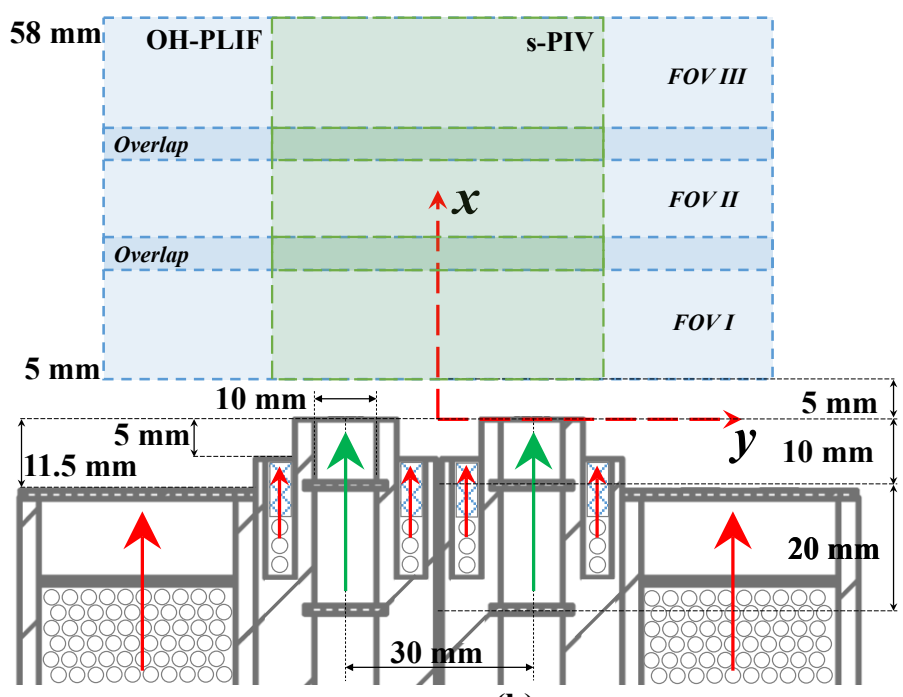

(b)

Figure 1: (a) Photograph of dual burner experiment in operation with all pilot flames. (b) Fields-ofviews (FOVs) for optical measurements; the red arrows represent the flow path for the pilot flame premixed gases and the green arrows represent the flow path for the main flame premixed gases

The test matrix used in this study is a subset of the datasets used in a previous study; definitions of each of the operational parameters can be found in Ref. [1]. For this work, three bulk flow velocities are used for both single- and dual-flame configurations (Table 1). Each flame condition is operated at $\phi=1.0$ and the flame spacing in the dual flames cases is kept constant at $30 \mathrm{~mm}$. All pilot flames are operated at $\phi=1.0$ to avoid gradients in product temperature or equivalence ratios. Bulk flow velocities of anchoring and back-support pilot flames are kept constant at $3 \mathrm{~m} / \mathrm{s}$ and $4.3 \mathrm{~m} / \mathrm{s}$, respectively. All main flames are operated in the thin-reactions regime on the BorghiPeters' premixed combustion regime diagram [20]. The turbulence intensity results presented in Table 1 are calculated using Equation (1):

$$
u^{\prime}=\sqrt{\left(\frac{u_{x}^{\prime 2}+u_{y}^{\prime 2}+u_{z}^{\prime 2}}{3}\right)}
$$


Table 1: Flow conditions of burners

\begin{tabular}{|c|c|c|c|c|c|c|c|c|c|c|c|}
\hline Case & $\begin{array}{c}\text { Bulk } \\
\boldsymbol{U} \\
{[\boldsymbol{m} / \boldsymbol{s}]}\end{array}$ & ow prop & $R e_{h}$ & $\begin{array}{c}\boldsymbol{u}^{\text {NoI }} \\
{[\boldsymbol{m} / \boldsymbol{s}]}\end{array}$ & $\begin{array}{c}\text { reacting } \\
\boldsymbol{L}_{\mathbf{1 1}} \\
{[\mathbf{m m}]}\end{array}$ & $\begin{array}{l}\text { inlet turl } \\
\lambda_{\text {Taylor }} \\
{[\mathbf{m m}]}\end{array}$ & $\begin{array}{l}\boldsymbol{R} \boldsymbol{e}_{\boldsymbol{L}_{1 \mathbf{1}}} \\
\end{array}$ & $\begin{array}{l}\text { haracter } \\
\boldsymbol{u}^{\prime} / \boldsymbol{s}_{\boldsymbol{L}}\end{array}$ & $\begin{array}{l}\text { stics } \\
L_{11} / l_{f}\end{array}$ & $\begin{array}{l}\text { Flame height } \\
\boldsymbol{H}[\boldsymbol{m m}]\end{array}$ & $\begin{array}{l}\text { Simultaneous } \\
\text { measurements }\end{array}$ \\
\hline $\begin{array}{l}\text { A-Dual } \\
\text { A-Single }\end{array}$ & 12 & 8500 & 15000 & 2.2 & 2.1 & 1.4 & 325 & 5.5 & 11.1 & $\begin{array}{l}35 \\
35 \\
\end{array}$ & $\begin{array}{l}\text { Yes } \\
\text { No }\end{array}$ \\
\hline $\begin{array}{c}\text { C-Dual } \\
\text { C-Single }\end{array}$ & 20 & 14000 & 26000 & 3.6 & 2.3 & 1.3 & 575 & 8.9 & 11.8 & $\begin{array}{l}48 \\
46 \\
\end{array}$ & $\begin{array}{l}\text { Yes } \\
\text { No }\end{array}$ \\
\hline $\begin{array}{c}\text { E-Dual } \\
\text { E-Single }\end{array}$ & 28 & 19000 & 36000 & 5.0 & 2.2 & 1.2 & 787 & 12.4 & 11.6 & $\begin{array}{l}58 \\
56 \\
\end{array}$ & $\begin{array}{l}\text { Yes } \\
\text { No }\end{array}$ \\
\hline
\end{tabular}

\subsection{Diagnostics and Data Processing}

\subsubsection{OH-Planar Laser Induced Fluorescence (OH-PLIF)}

Flame-front measurements are performed using $\mathrm{OH}$-planar laser-induced fluorescence $(\mathrm{OH}-$ PLIF) at a sampling rate of $10 \mathrm{kHz}$. The OH-PLIF system consists of a $532 \mathrm{~nm} \mathrm{Nd:YAG} \mathrm{laser}$ (Edgewave) pumping a dye laser (Sirah Credo). The output beam is tuned to the Q1(6) line of the $\mathrm{A}^{2} \Sigma^{+} \leftarrow \mathrm{X}^{2} \Pi(1-0)$ band to excite the $\mathrm{OH}$ radicals at $282.94 \mathrm{~nm}$. The UV beam from the dye laser is passed through a periscope and a set of three cylindrical lenses to obtain a collimated sheet with an approximate height of $21 \mathrm{~mm}$. The signal from the excited $\mathrm{OH}$ radicals is acquired using a CMOS sensor camera (Photron FASTCAM SA1.1), coupled with an external intensifier (LaVision HS-IRO) and a $100 \mathrm{~mm} \mathrm{f} / 2.8 \mathrm{UV}$ lens (Cerco), resulting in a resolution of $0.1 \mathrm{~mm} / \mathrm{pixel}$. A high transmissivity filter (LaVision $1108760 \mathrm{VZ}$ ) is used to collect the signal at $320 \pm 20 \mathrm{~nm}$. Background flame luminosity is reduced in the acquired images by setting the intensifier gate at 100-150 ns. Simultaneous OH-PLIF/s-PIV measurements datasets include 5000 images and 10000 images for only OH-PLIF datasets. Measurements are performed in three FOVs with a $5 \mathrm{~mm}$ overlap between two FOVs. The signal to noise ratio of the images is $\sim 32$ based on the methodology described in [2]; further discussion of uncertainty associated with PLIF imaging acquisition and processing is discussed in the supplemental material of our previous work [1]. 


\subsubsection{Stereoscopic-Particle Image Velocimetry (s-PIV)}

Stereoscopic-particle image velocimetry is performed at $10 \mathrm{kHz}$ with a dual cavity, Nd:YAG laser (Quantronix Hawk Duo) operating at $532 \mathrm{~nm}$ in forward-forward scatter mode. A $50 \mathrm{~mm}$ tall laser sheet is created using a combination of mirrors and three cylindrical lenses; the angle between the laser sheet plane and each camera sensor (Photron FASTCAM SA5) is about 25 degrees. Each camera is equipped with a $100 \mathrm{~mm} \mathrm{f} / 2.8$ lens (Tokina Macro) and a Nikon tele-converter to allow for a safe stand-off distance between the sensor and the burners. A $32 \mathrm{~mm}$ x $53 \mathrm{~mm}$ field of view is obtained through this setup and images are collected at $10 \mathrm{kHz}$ in double frame mode with a pulse separation of $14 \mu \mathrm{s}$. Aluminum oxide particles of diameters $0.5-2.0 \mu \mathrm{m}$ are used for seeding the flow field; the Stokes number based on a $1 \mu \mathrm{m}$ nominal diameter of these particles is 0.06 , implying that particles can track flow oscillations up to $4000 \mathrm{~Hz}$ [21]. To reduce flame luminosity, near-infrared filters (Schneider Kreuznach IR MTD) and laser line filters (Edmund Optics TECHSPEC $532 \mathrm{~nm}$ CWL) are used on each camera. LaVision's DaVis 8.3 is used to perform vector calculations from Mie scattering images. These calculations included a multi-pass algorithm with varying window sizes ranging from $64 \times 64$ to $16 \times 16$ and a 50\% overlap. This resulted in a vector spacing of $0.48 \mathrm{~mm} /$ vector. A universal outlier detection scheme, with a $3 \mathrm{x}$ median filter is used for post-processing of the vector fields. Averaged uncertainties in instantaneous velocities range from $1.4-2.5 \mathrm{~m} / \mathrm{s}$ in the jet region of the burners for $U=12-28 \mathrm{~m} / \mathrm{s}$ using the uncertainty calculation feature in DaVis. A total of 5000 vector fields are obtained for each condition. The layout of the simultaneous OH-PLIF and s-PIV system is shown in Ref. [1]. Stanford Research Systems DG-535 digital delay generators are used to synchronize the OH-PLIF and s-PIV systems.

\subsubsection{Pocket Identification and Tracking}


One of the necessities of flame pocket identification is accurate binarization of raw OH-PLIF images. In this study, a multi-step binarization scheme is implemented: 1) laser-sheet profile corrections are applied to the images, 2) corrected images are median- and bilateral-filtered to smooth discrete intensity changes that may result in noise while preserving the $\mathrm{OH}$-gradients corresponding to flame fronts, 3) dynamic thresholding is performed using Otsu's method [22] to obtain binarized images, and 4) flame edges are identified by tracing the binarized objects from the previous step. Sensitivity of our analysis to binarization parameters was quantified and reported in the supplemental material of Tyagi et al. [1].

Pockets can be identified using the flame edges calculated from the binarized images. The bwboundaries algorithm stores each non-connected edge in each frame as a separate object in a cell array, allowing for identification of a pocket edge from the main flame. Flame edges that contain 'islands' of reactants or products are flagged and the binarized pixel values contained in the islands are utilized to discern reactant islands from product islands. Reactant pockets are islands with values of zero inside, and product pockets are islands with values of one inside. Geometric properties, such as contour centroid, area, perimeter, etc., are also stored along with the edges. An example of the binarization scheme and identified islands are shown in Figure 2.
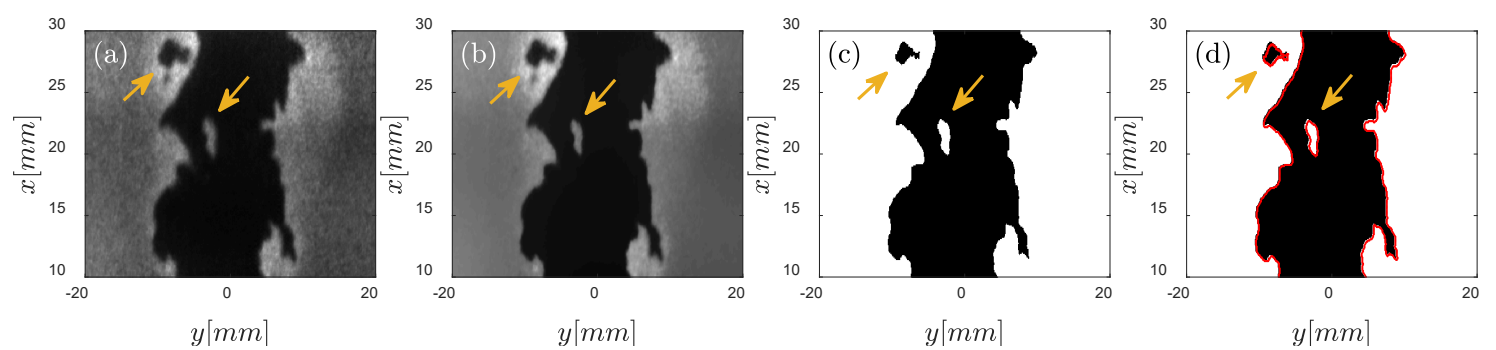

Figure 2: (a) Sheet-corrected OH-PLIF image, (b) bilateral and gaussian filtered image, (c) binarized image, and (d) binarized image with edges 
This technique works well for identifying islands of reactants and products; however, to classify these islands as actual 'pockets' requires some assumptions. Pockets formed in turbulent flames are three dimensional and the use of planar imaging limits full identification of the flame surface of the pocket. Without the knowledge of the complete flame surface in three dimensions, uncertainties are introduced by treating detected islands as individual pockets. Reactant pockets are commonly observed in flames operating in thin-reactions regimes and, as such, we assume that the reactant islands identified in this study are likely reactant pockets [20]. In our previous work [1], we quantified the likelihood that a reactant-side interaction is an actual interaction rather than an out-of-plane motion. To determine this, we assumed that if the local out-of-plane velocity, measured using s-PIV, was higher than a turbulent flame speed $\left(S_{T}=\sqrt{1+\left(u^{\prime} / s_{L}\right)^{2}}\right)$, then the interaction was likely to be an out-of-plane motion rather than an actual interaction. Otherwise, we assumed that the interaction was real. For Case A, the likelihood that the pocket-forming interaction is real is $92 \%$, whereas for Case E, $82 \%$ interactions are real.

In the case of islands containing products, it is uncertain whether to treat the identified islands as isolated pockets or connected tunnels that exist in the out-of-plane direction [6]. While it is possible to capture the out-of-plane surface using tomographic imaging methods [23-26], it becomes rather expensive to perform such measurements and they include a new set of uncertainties [27].
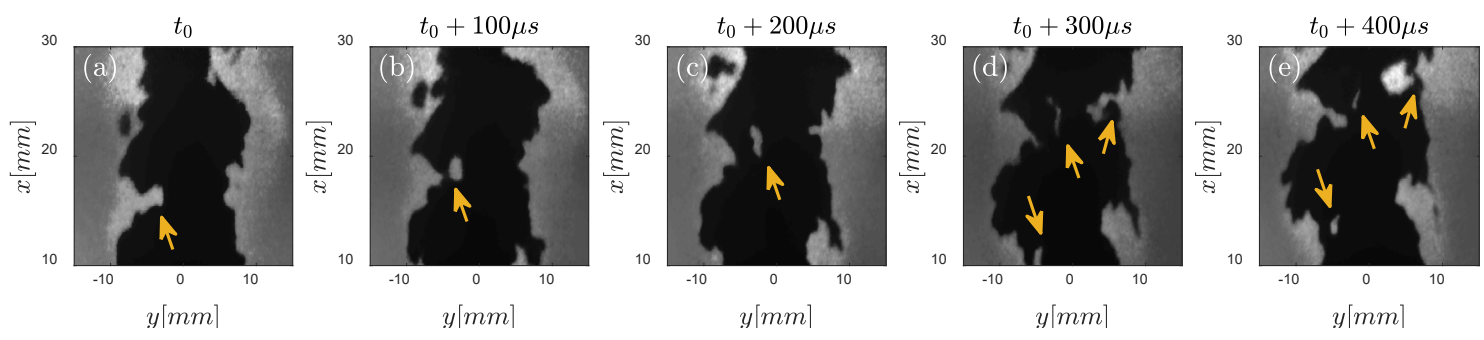

Figure 3: Example of product islands identified in OH-PLIF images 
Figure 3 shows examples of product islands in case E-Single; sub-figures (a)-(e) show an example of merging of product sides of the flame front, resulting in the formation of a product island, as shown in sub-figure (b). This island convects downstream and appears to shrink in the plane of measurement, as shown in sub-figures (c), (d), and (e). In the last two sub-figures, two other product islands are formed. These instances of product islands are merely examples of the different ways these islands are formed in turbulent flames. While classifying these islands as product pockets or tunnels is a limitation of the planar measurements, we report results assuming these product 'islands' to be product pockets. Simulation studies of flame-flame interactions have shown that tunnel formation rates are generally higher than product pocket formations in turbulent premixed flame $[6,28,29]$. This difference in rates could mean that most of the identified product islands are results of tunnel formations perpendicular to the measurement plane. While these challenges prevent us from providing conclusive results on product pockets, they still describe mechanisms affecting the flame surface generation and destruction. We expand more on the effects of both product pockets and product tunnels on local flame propagation in Section 3.3, but until then, report these islands as pockets in our statistics in Section 3.1-3.2.

Once the detected pocket information is stored, a detailed tracking scheme is implemented to capture the lifetime of these pockets: 1) pockets detected in consecutive images are grouped together, 2) for each pocket, a search is conducted for pockets in the next frame that correspond to the current pocket of interest using a nearest distance approach with a threshold convection distance based on the bulk flow velocity and the velocity fluctuations, and 3) if a pocket is identified in the next frame, a pocket number is assigned to the pocket of interest and the identified pocket. These steps are repeated for all the pockets in the group until all pockets are assigned a pocket number. Pocket numbers are updated if pocket tracking halts in consecutive frames. To 
ensure that pockets are correctly tracked, the alignment of the trajectory of pockets with the convecting direction of the flow field is checked at every step.

\subsubsection{Pocket Origin and Fate Identification}

Non-rigid image registration methods are implemented on consecutive binarized image to identify a pocket's origin and fate. This method estimates non-uniform displacement fields to match features present in two consecutive images. A detailed discussion of this technique is beyond the scope of this paper and more details can be found in [30, 31]. In previous work [1], we used this method to rigorously identify topological differences in the flame surface that occur during flame interactions; we use this same method to identify the original interaction in which each pocket is formed. Uncertainty analysis associated with this non-rigid image registration technique is detailed in the supplemental material of Ref. [1]. In this study, consecutive binarized images (called 'fixed' image at $t=t_{0}$ and 'moving' image at $t=t_{0}+\Delta t$ ) are registered and flame edges of the registered images are utilized to check whether the pockets originate from flameflame interactions observed in the plane of measurements or if they appear from out-of-plane. Edges of flame pockets from the registered moving image are checked with the edges of flame fronts in the fixed image. Intersection of the pocket edges with the flame front edges would indicate that flame pockets originate from flame-flame interactions. For no intersections, the pockets are assumed to appear from out-of-plane. Finally, pockets that appear from the bottom of the FOV are

assumed to convect into the FOV. In this study, these three types of origin of flame pockets are referred to as "Interaction", “Appear", and "From bottom”, respectively. 

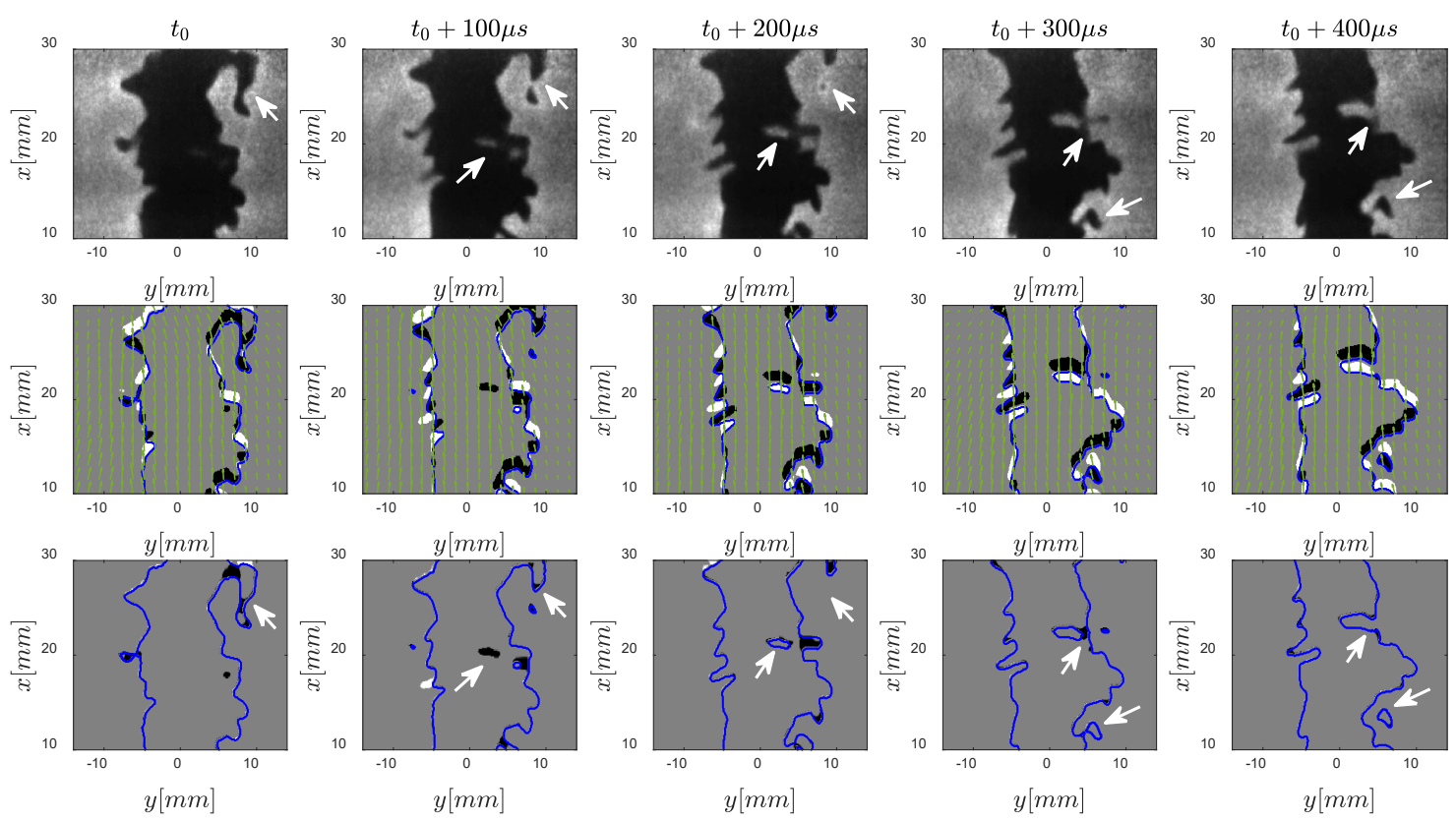

Figure 4: The use of image registration on tracking the origin and fate of reactant and product pockets

An approach similar to pocket origin identification is implemented for categorizing what happens at the end of the measurable pocket lifetime. Pockets that merge with the main flame front are referred to as "Merged" and merging events are identified with the same non-rigid image registration technique used for interaction identification. Pockets that convect out the top of the FOV are referred to as "Moved out". Finally, pockets that disappear within the FOV are referred to as "Consumed/Disappeared", where the category "Consumed" is used for reactant pockets and "Disappeared" used for product pockets. Examples of origin and fate of flame pockets are shown in Figure 4; the top row shows a time-series of raw OH-PLIF images, while the bottom row shows a series of moving registered binarized images subtracted from fixed binarized images with edges extracted from the fixed edges. In these images, the blue boundaries represent the flame edges, the gray color represents regions with no changes between fixed and moving images, and white and back regions represent changes due to topological changes on the flame surface. Green vector 
fields represent the down-sampled displacement fields generated from registration. The arrows in this figure highlight the origin and fate of reactant and product pockets.

\section{Results and Discussions}

\subsection{Global effects}

Figure 5 (a) shows the stitched flame surface density (FSD) of case A-Single from all three FOVs. Flame surface density is calculated using an interrogation window size of 1 x 1 pixel with a pixel resolution of $0.1 \mathrm{~mm} /$ pixel. Flame edges are counted in these interrogation windows for 10,000 images and the average signal in each interrogation window is divided by the window area to obtain FSD. This image shows that FSD is high at the base of the flame and decreases with downstream distance as the flame brush thickness increases. The impact of flame pockets on the time-averaged flame structure is quantified by calculating the FSD neglecting the surface contribution from the pockets. This new FSD is subtracted from the total FSD to quantify the impact that flame pockets have on global flame structure. These results are shown in Figure 5 (b)(d) for case A-single and results for the other cases are shown in Figures S1-S6 in the supplemental material; the colorbar in all figures is adjusted to highlight the FSD differences in these images. Figure 5 (b) shows the difference in FSD with all the pockets subtracted, whereas Figure 5 (c) shows the difference in FSD with just the reactant pockets subtracted. These images are similar, indicating that the pockets do not have a large effect on FSD near the base of the flame but contribute a more significant portion where flame tip pinching is present. Similar spatial distributions of reactant pocket formation and burnout were observed by Worth and Dawson [32], showing that reactant pockets are more likely to form near the tip. Figure 5 (d) shows the difference due to the lack of product pockets, where product pockets are more likely to form near the base of 
the flame and do not account for a significant portion of the FSD. Altogether, these results indicate that flame pockets contribute to $10-20 \%$ of the total flame surface, depending on operating condition.
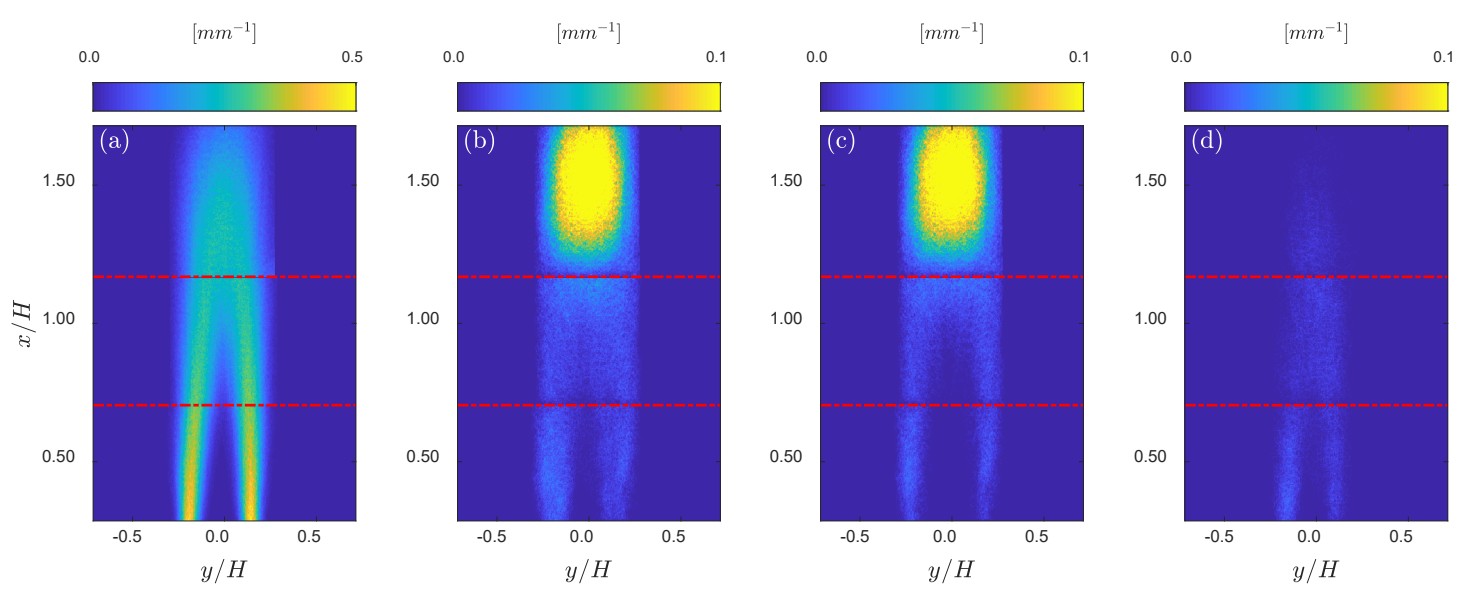

Figure 5: Flame surface density contributions for flame A-Single: (a) total, (b) all pocket contributions, (c) reactant pocket contributions, and (d) product pocket contributions

Figure 6 shows horizontal slices of the FSD and FSD differences shown in the contours in Figure 5 for a range of turbulence intensities; note the difference in y-axis scales between the full FSD and the FSD differences. Data is compared at several normalized downstream locations, $x / H=0.25-1$, but not further downstream due to limitations of the FOV at the highest turbulence intensity (Case E). These cuts more clearly show the difference in the impact of pockets on the FSD as a function of turbulence intensity. Near the burner exit region, shown in Figure 6 (a), there are measurable differences in the pocket contribution as turbulence level is varied. Pocket contributions in flames A are smaller compared to flames E; increased turbulence levels in flames E can result in frequent flame pinch-off, resulting in increased pocket formations. These differences due to turbulence level are minimal further downstream, as in Figure 6 (d), because the inlet turbulence levels have decayed to similar levels in all cases; see Figure 12 (d) for turbulence intensities at the $\bar{c}=0.5$ contour, representative of the turbulence at the flame front. Additionally, 
there is almost no difference between the trends of the single- and dual-flame configurations at each turbulence intensity, which indicates that the large-scale interaction between the two flames and flow fields does not impact the small-scale pocket formation in the Bunsen flame configuration.
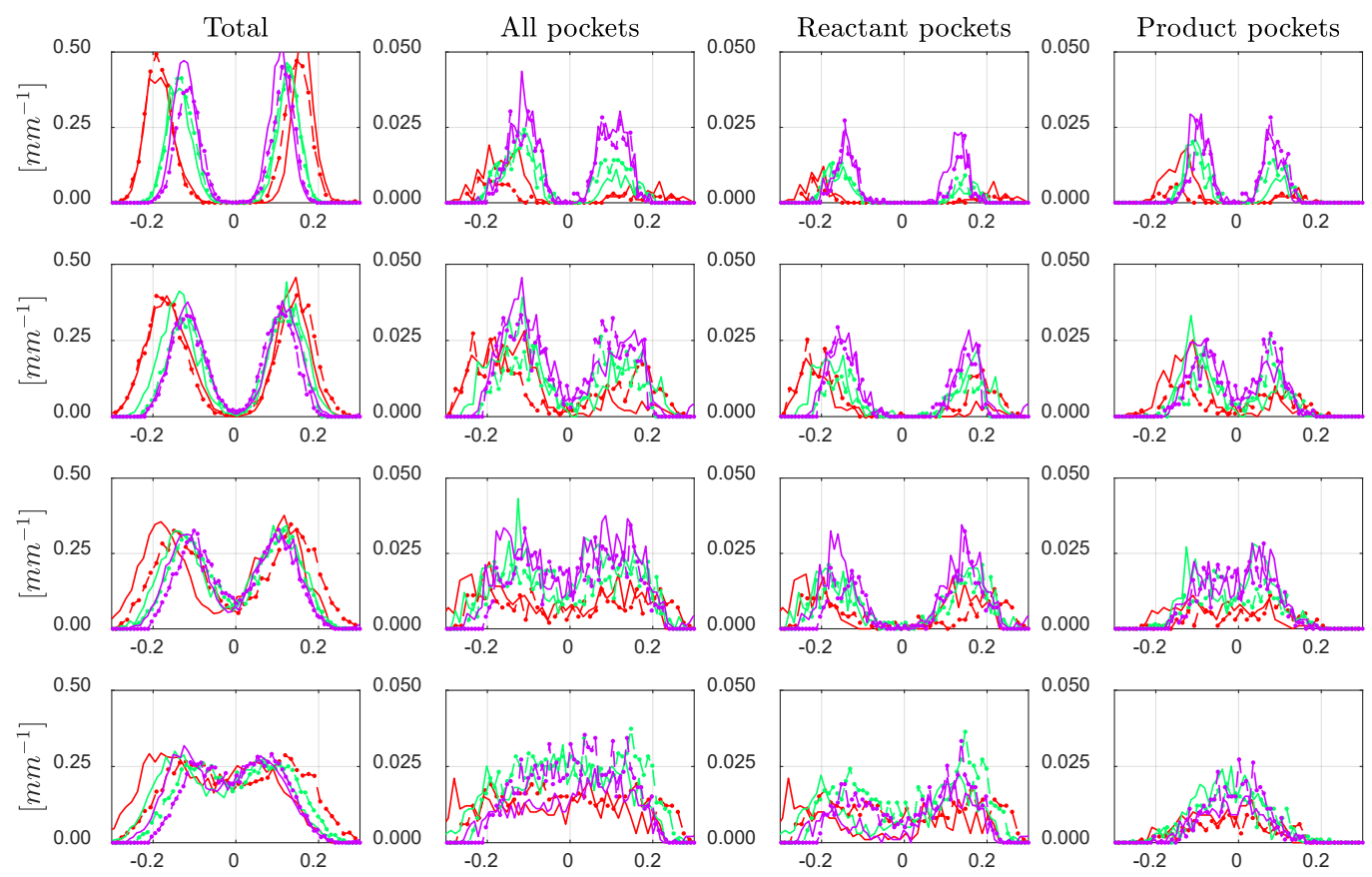

$y / H$

$$
y / H
$$

Figure 6: Horizontal slices of flame surface density contributions at various downstream locations: (a) $x / H=0.25$, (b) $x / H=0.5$, (c) $x / H=0.75$, and (d) $x / H=1$. For comparisons between single- and dual-flames, the $y$-axis is adjusted to the left burner centerline in dual-flames cases. For clarity, every fifth data point is plotted here

Histograms of the original size of each pocket are shown in Figure 7; the size is quantified as the mean radius of the pocket, which is calculated by first identifying the center of mass of the pocket (assuming constant density inside) and then averaging the radii along the length of the pocket perimeter. The integral length scale and the Taylor microscale, calculated at the exit of the burner, are provided for reference in each case. In the first two FOV, reactant and product pockets have similar size distributions. In FOV III, the reactant pocket distribution has a tail of larger radius 
pockets that are indicative of the pinch-off of large pockets at the tip of the flame. In general, most of the pockets are smaller than both the integral length scale and the Taylor microscale, indicating that these pocket formations may be occurring on the sub-grid in many large-eddy simulations (LES). Despite their small size, we show in the next section that these pockets are formed quite frequently, making the dynamics of these pockets a significant contributor to local flame surface dynamics.
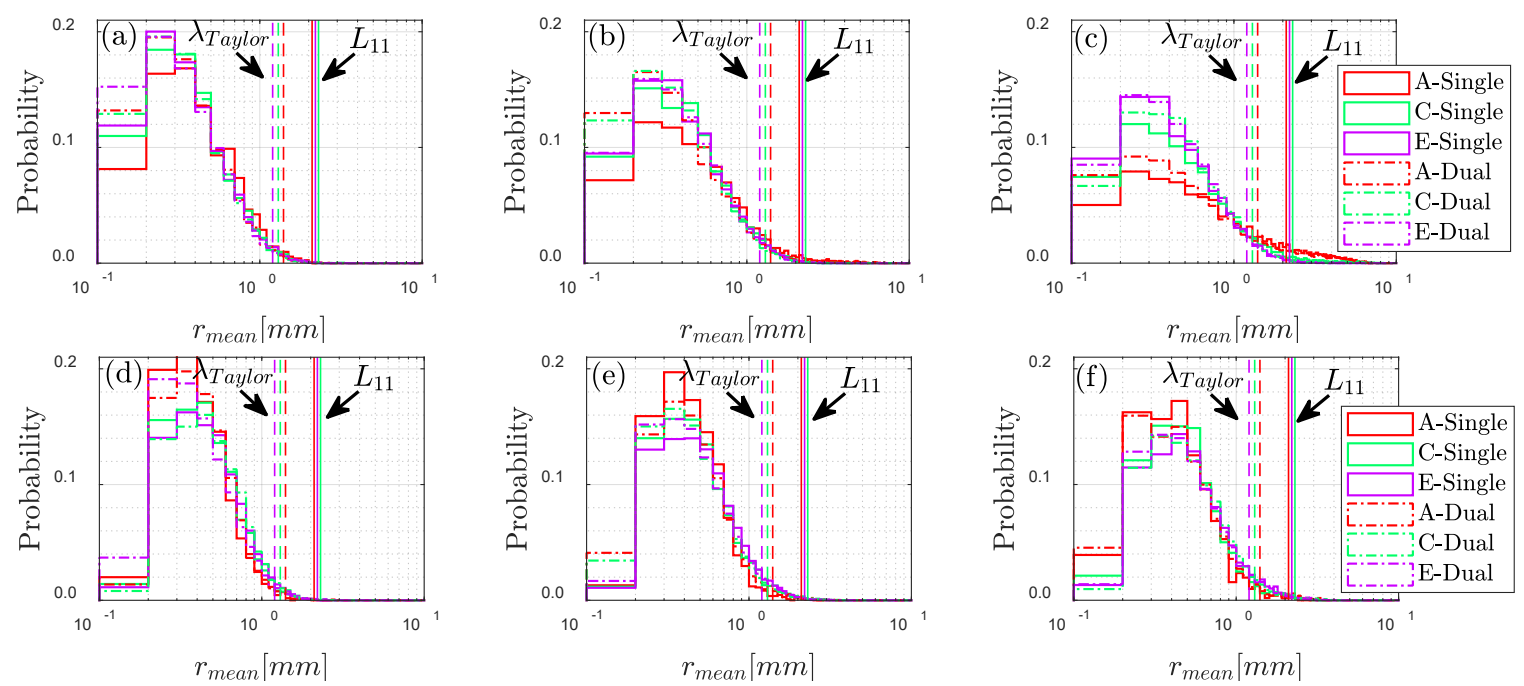

Figure 7: Mean pocket radii for reactant pockets in (a) FOV I, (b) FOV II, and (c) FOV III. Mean pocket radii for product pockets in (d) FOV I, (e) FOV II, and (f) FOV III

\subsection{Pocket origin}

Figure 8 shows the formation rate of reactant and product pockets $\left(\mathcal{R}_{R, p}\right.$ and $\left.\mathcal{R}_{P, p}\right)$ in units of Hertz for both single and dual flames. In each sub-figure, the rate is calculated per FOV and plotted against the mid-point of the vertical location of the FOV normalized by the flame height. The flame height is calculated based on the height of the time-averaged progress variable contour of $\bar{c}=0.2$. Additionally, the results plotted in these sub-figures for dual flames cases are averaged between the two flames to make direct comparisons with single-flame cases. Uncertainty in the identification of pocket formation events was quantified in our previous work [1]; we expect that 
as high as $98 \%$ (in low turbulence cases) and as low as $81 \%$ (in high turbulence cases) of the identified interactions are the result of in-plane motion as opposed to out-of-plane motion.
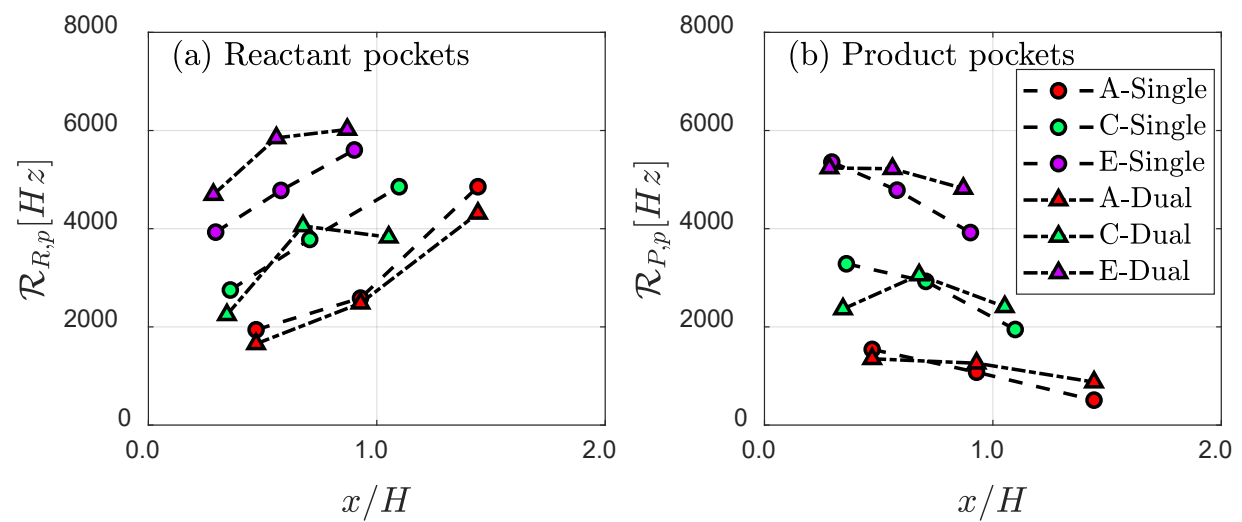

Figure 8: Frequency of pocket formations in [Hz] for: (a) reactant pockets, (b) product pockets

Reactant pocket formation rates (Figure 8 (a)) increase with downstream distance for both single- and dual-flames cases. As the bulk flow velocity increases, the rate of reactant pocket formation also increases, indicating that higher turbulence levels increase wrinkling of the flame front, resulting in increased tendency of the flame to pinch-off and form pockets of reactant gases. These findings are congruent with the FSD plots shown in Figure 5. For cases A-Single and ADual, an abrupt increase in the reactant pocket formation is seen in FOV III (corresponding to $x / H=1.4)$. This FOV captures the flame tip region for these flames and more reactant pockets are observed to be formed in this region due to flame pinch-off, as shown by Figure 5 (b).

The product pocket formation rates presented in Figure 8 (b) show that product pockets tend to form in the upstream regions of the flame. Product pocket formation is more frequent when large strain rates are present on the reactant side of the flame surface; this strain can dominate flame propagation and result in splitting of product gases from the main flame $[6,33]$. The increase in the bulk flow velocity also increases the turbulent fluctuation intensity and high strain rates can be present in these flames, resulting in increased product pocket formation for cases $\mathrm{C}$ and $\mathrm{E}$ (in 
both single- and dual-flames). Additionally, as the turbulence level decreases with downstream distance (see Figure S11 in the supplementary material), the product pocket formation rates also decrease; note that these trends may be less significant given the up to $20 \%$ uncertainty associated with identifying interaction events. Trends for reactant and product pocket formations as a function of downstream distance shown in Figure 8 (b) match well with the FSD plots shown in Figure 5. Comparisons between single- and dual-flame cases show that pocket formation rates are very similar for both configurations. These pocket formation rates closely follow the interaction rates reported in our previous work [1], where reactant-side interactions increase frequency with downstream distance and product-side interactions decrease frequency with downstream distance.
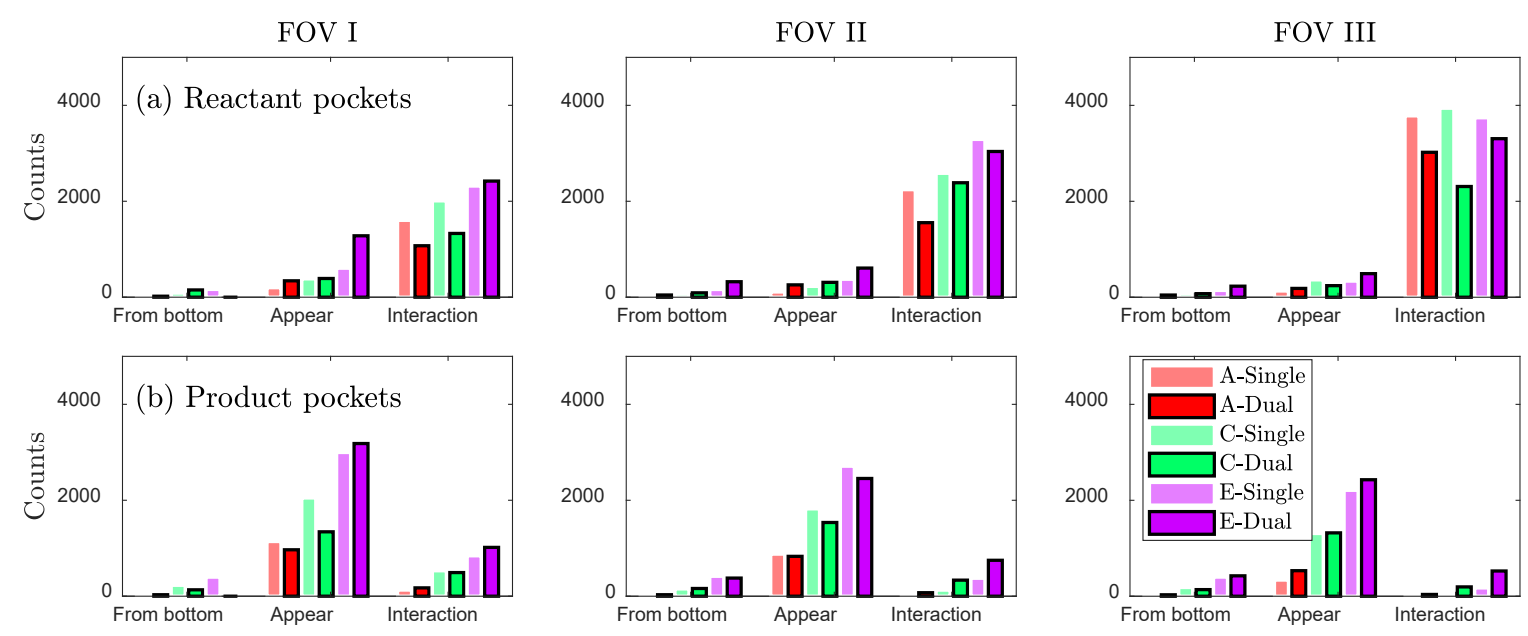

Figure 9: Statistics of pocket origin: (a) reactant pockets, (b) product pockets in the measurement plane

The origin of flame pockets is identified using the scheme outlined in Section 2.2.4. Figure 9 shows the origin of reactant and product pockets for single and dual flames in FOVs I-III. Reactant pockets are most likely to originate from flame-flame interaction events and very few reactant pockets appear from out-of-plane. Additionally, the probability of reactant pockets being formed from observed flame-flame interactions increases as the downstream distance increases along the flame, which aligns with the reactant pocket formation rate results (Figure 8). The majority of the 
product pockets appear from out-of-plane and very few pockets originate from flame-flame interactions observed within the plane of measurement (Figure 9 (b)). The fact that most reactant pockets originate from interaction sites provides further confidence that the detected pockets are, indeed, actual pockets and not three-dimensional motions. The fact that most product pockets appear from out of plane, and not from interaction sites, is the reason for our lower confidence in identifying these product islands as product pockets. However, there is currently no method for separating these two types of product islands in a planar measurement and so we continue to analyze them as both pockets and through-plane events, as will be discussed in Section 3.3.

\subsection{Pocket fate}

The local impact of pockets on the main flame can be captured by tracking the fate of pockets. For example, pockets can either burn out or merge with the main flame, locally altering the consumption of reactants and the local topology of the flame. Figure 10 (a) shows the statistics of reactant pocket fate in FOVs I-III. Results show that most reactant pockets burn out; a smaller number of pockets move out of the FOV and their fate remains unknown. A small number of reactant pockets merge with the main flame surface. Merging of reactant pockets with the main flame surface can result in perturbations to the flame surface area and consequently to the local heat release rate. Increasing the bulk flow velocity also increases the frequency of reactant pocket formation, resulting in increasing frequency of all pocket fates by a similar fraction. Note that the size of the FOV affects the balance of these histograms for both pocket origin and fate; a larger field of view would result in fewer "moved out" pockets, for example. 

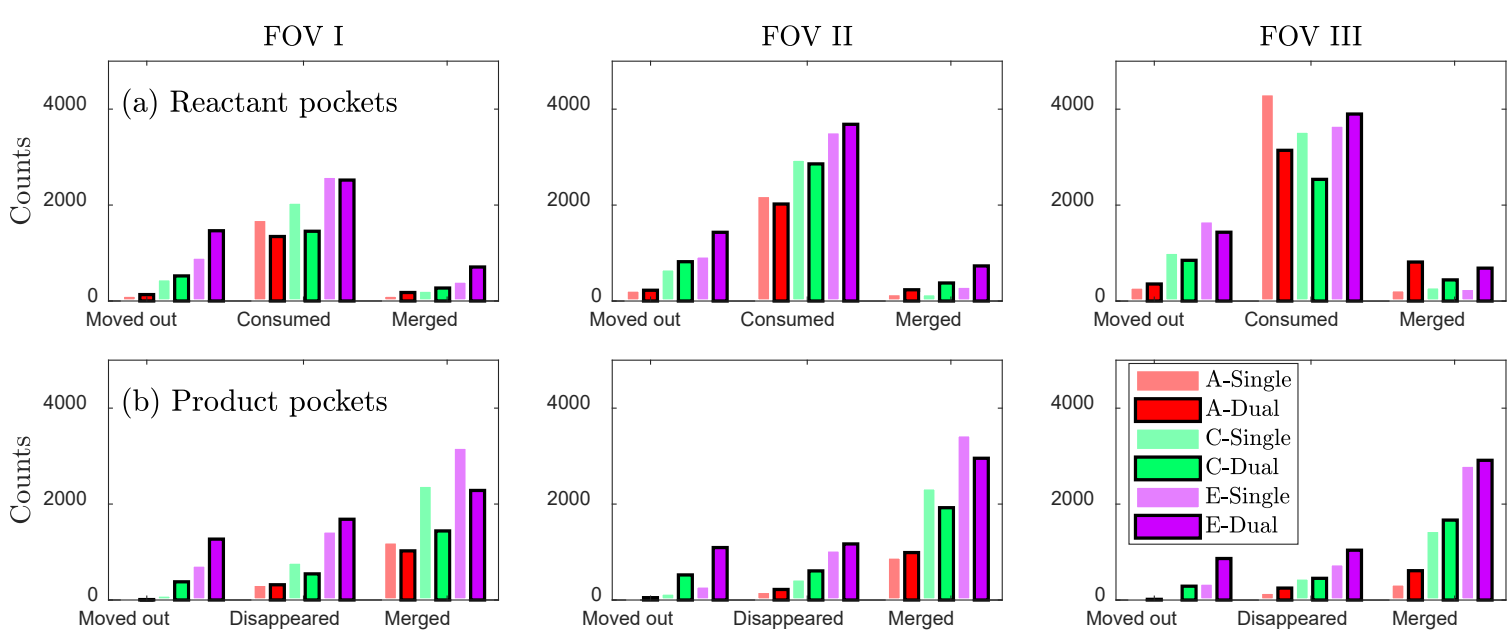

Figure 10: Statistics of pocket fate: (a) reactant pockets, (b) product pockets in the measurement plane

Next, as the fate of most reactant pockets is burnout, we quantify the pocket burnout rate using a local displacement speed. We implement the displacement speed formulation followed by Trunk et al. [34] and Peterson et al. [35] within the measurement plane to obtain an in-plane flame displacement speed:

$$
S_{D}=\left|\vec{u}_{\text {pocket }}-\vec{u}_{\text {conv }}\right|
$$

In Equation (2), $\vec{u}_{\text {pocket }}$ is the velocity of the reactant pocket flame surface, $\vec{u}_{\text {conv }}$ is the flow convection velocity, and $S_{D}$ is the local flame displacement speed. Displacement vector fields from the image registration analysis carried out in this study (described in Section 2.2.4) are utilized to calculate the total reactant pocket motion $\left(\vec{u}_{\text {pocket }}\right)$ in a time interval $\Delta t$. The image registration technique provides a displacement vector field for the surface between time $t_{0}$ and $t_{0}+\Delta t$; dividing that displacement vector by the interframe time provides $\vec{u}_{\text {pocket }}$. Simultaneously measured velocity fields are used to obtain the local convection velocity $\left(\vec{u}_{\text {conv }}\right)$ along the reactant pocket contour and the reactant pocket displacement speed $\left(S_{D}\right)$ is obtained. Due to the differences in resolutions between OH-PLIF images and the velocity fields, $S_{D}$ is only calculated along the 
reactant pocket contour coordinates that align with the velocity field pixel locations. This precautionary step is carried out to avoid duplicating calculated values of $S_{D}$ that can lead to incorrect interpretation of the results.

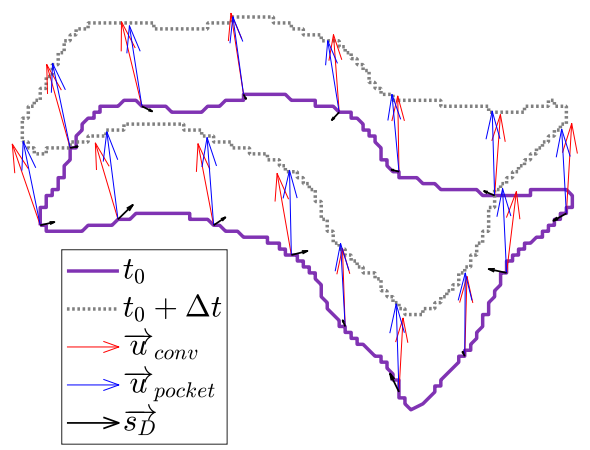

Figure 11: Calculating $s_{D}$ from displacement fields and velocity fields

Figure 12 (a)-(c) shows the PDFs of $S_{D}$ for cases Dual-A, C, and E in FOVs I-III. In each of these subfigures, the corresponding inlet $u^{\prime}$ velocity for each case is marked with a colored vertical line to compare with peaks of the PDF plots. Results here show that as the bulk flow velocity is increased, the PDF distribution becomes very wide, indicating that turbulence affects the reactant pocket displacement, especially in FOV I for case E-Dual. Within this FOV, the peaks of $S_{D}$ distributions for all cases are present near their corresponding inlet $u^{\prime}$ values at the burner exit, suggesting that local turbulence directly impacts the most probable pocket displacement speed. As the downstream distance is increased (FOVs II and III), the PDF shapes become narrower compared to their shapes for FOV I.

It is interesting to note that the peak $S_{D}$ also decreases with downstream distance. Figure 12 (d) plots the fluctuating components of velocity $\left(u_{x}^{\prime}, u_{y}^{\prime}\right.$, and $\left.u_{z}^{\prime}\right)$ along the $\bar{c}=0.5$ contour as a function of normalized downstream distance. The turbulence level along the $\bar{c}=0.5$ contour decays with downstream distance and the fluctuating velocity $u_{x}^{\prime}$ reaches a value in the range of $1-2 \mathrm{~m} / \mathrm{s}$ 
for dual flames A, C, and E in FOV III. As these velocity component magnitudes decay to very similar values for all cases in FOV III, the turbulent level in the flow field does not significantly alter the most probable value for $S_{D}$, which can explain why the PDF peaks for all cases are very similar in this region of the flame.
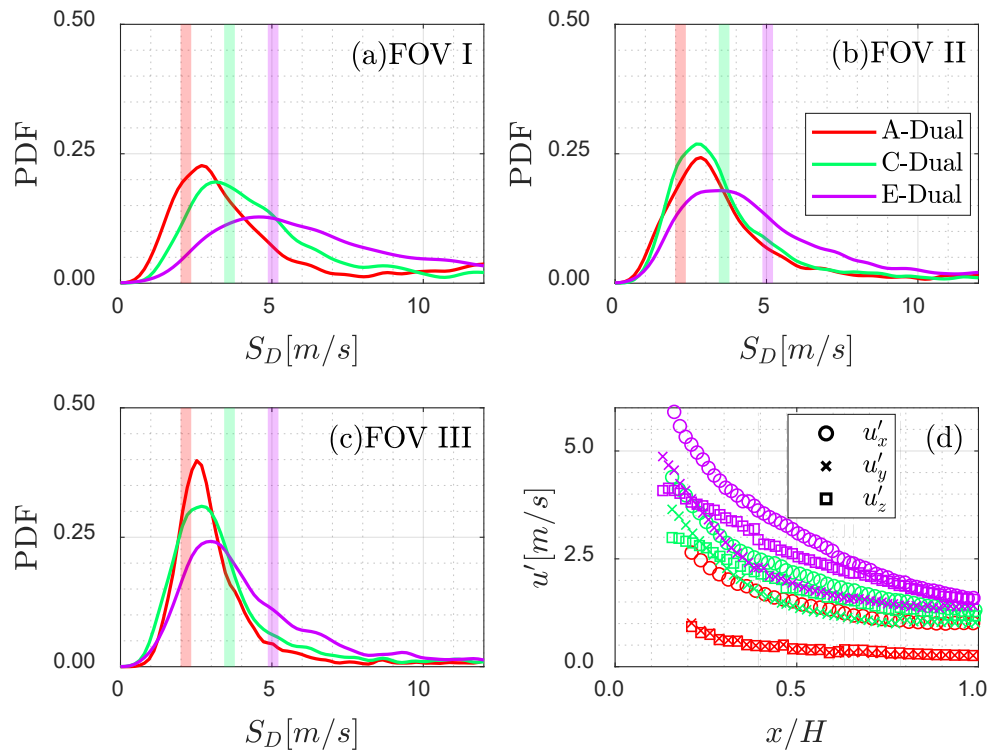

Figure 12: PDFs of reactant pocket displacement speeds: (a) FOV I, (b) FOV II, and (c) FOV III. Vertical colored lines represent $S_{D}=u^{\prime}: 2.2 \mathrm{~m} / \mathrm{s}$ for A-Dual, $3.6 \mathrm{~m} / \mathrm{s}$ for C-Dual, and $5.0 \mathrm{~m} / \mathrm{s}$ for E-Dual at the burner exit location. (d) Turbulence intensities along $\bar{c}=0.5$ as functions of downstream distance for cases Dual-A, $\mathrm{C}$, and $\mathrm{E}$

The fate of product pockets is quite different than that of reactant pockets. As mentioned in Section 2.2.3, it is challenging to distinguish product tunnel formations from product pockets using planar imaging techniques. Figure 13 shows two examples of product pockets in case A-Single; in sub-figure (a), the product pocket is formed near the bottom of the FOV, increases in size as it convects downstream, and merges with the flame front, resulting in a flame surface addition event. This particular example shows that pocket shapes change over time once they are formed and merging of these products with the main flame can result in changes in the local flame structure. 

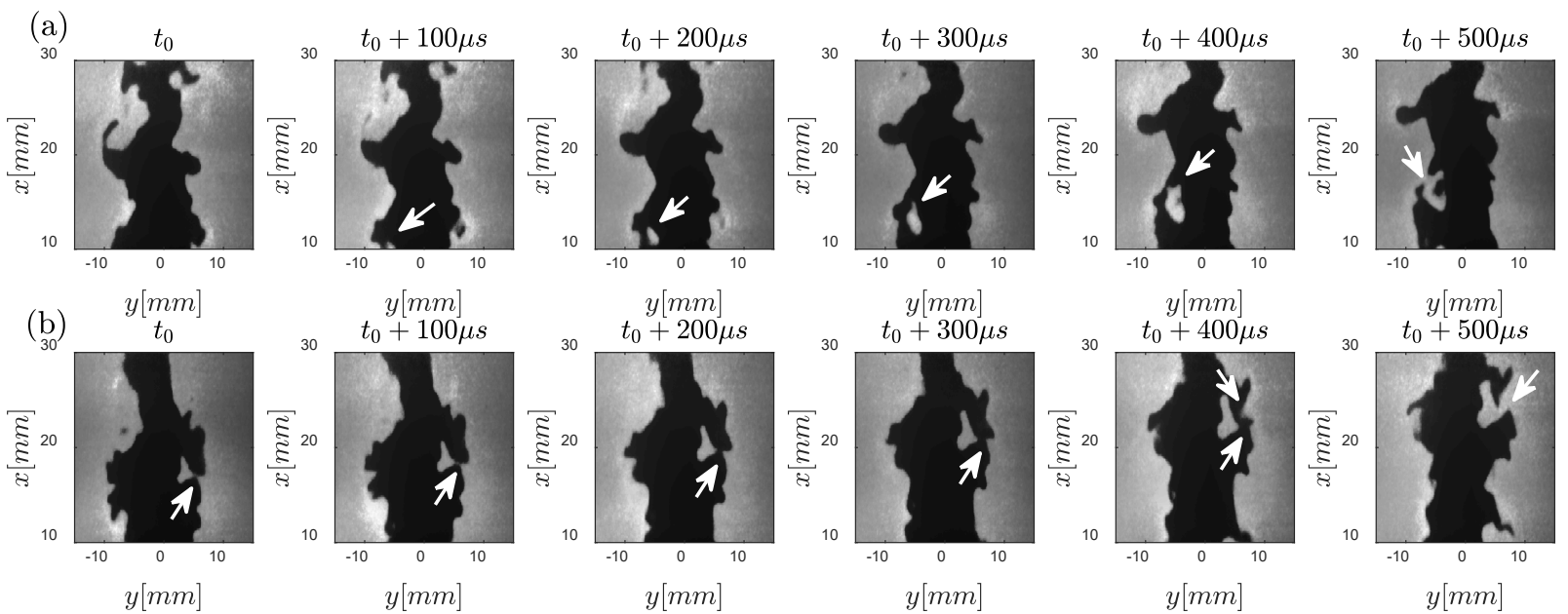

Figure 13: Examples of time-series of product pockets in flame A-Single

In sub-figure (b), another product pocket time-series is shown to highlight how these identified structures appear to be pockets but are likely just flame tunnels. Since its inception, the product pocket remains closer to the main flame surface and eventually merges with the flame surface. While this process is similar to that shown in Figure 13 (a), the appearance of this pocket fits more closely with a tunnel-like structure given its constant proximity to the main flame surface. Simultaneous velocity fields can be utilized to quantify the averaged out-of-plane velocity component within these product gas structures in an attempt to distinguish between product tunnels and product pockets. Figure 14 shows histograms of the averaged out-of-plane velocities contained within product contours for dual flames A, C, and E in FOVs I-III. In each sub-figure, colored vertical lines represent relevant flame velocity scales: the laminar flame speed, the maximum flame speed before stretch-induced extinction, and the turbulent flame speed. For the turbulent flame speed $\left(S_{T}\right)$, an uncertainty $\left(\delta S_{T}\right)$ is calculated based on uncertainties on $u^{\prime}$ from PIV measurements; details of the uncertainty calculation can be found in the supplementary material of Ref. [1]. The probability of out-of-component velocity instances lower than these flame speed metrics are shown in each plot. While the laminar flame speed and the flame extinction velocity 
do not scale with increasing turbulence level, these metrics quantify the magnitude of out-of-plane motion, which informs our interpretation of the product pockets in the LIF images.
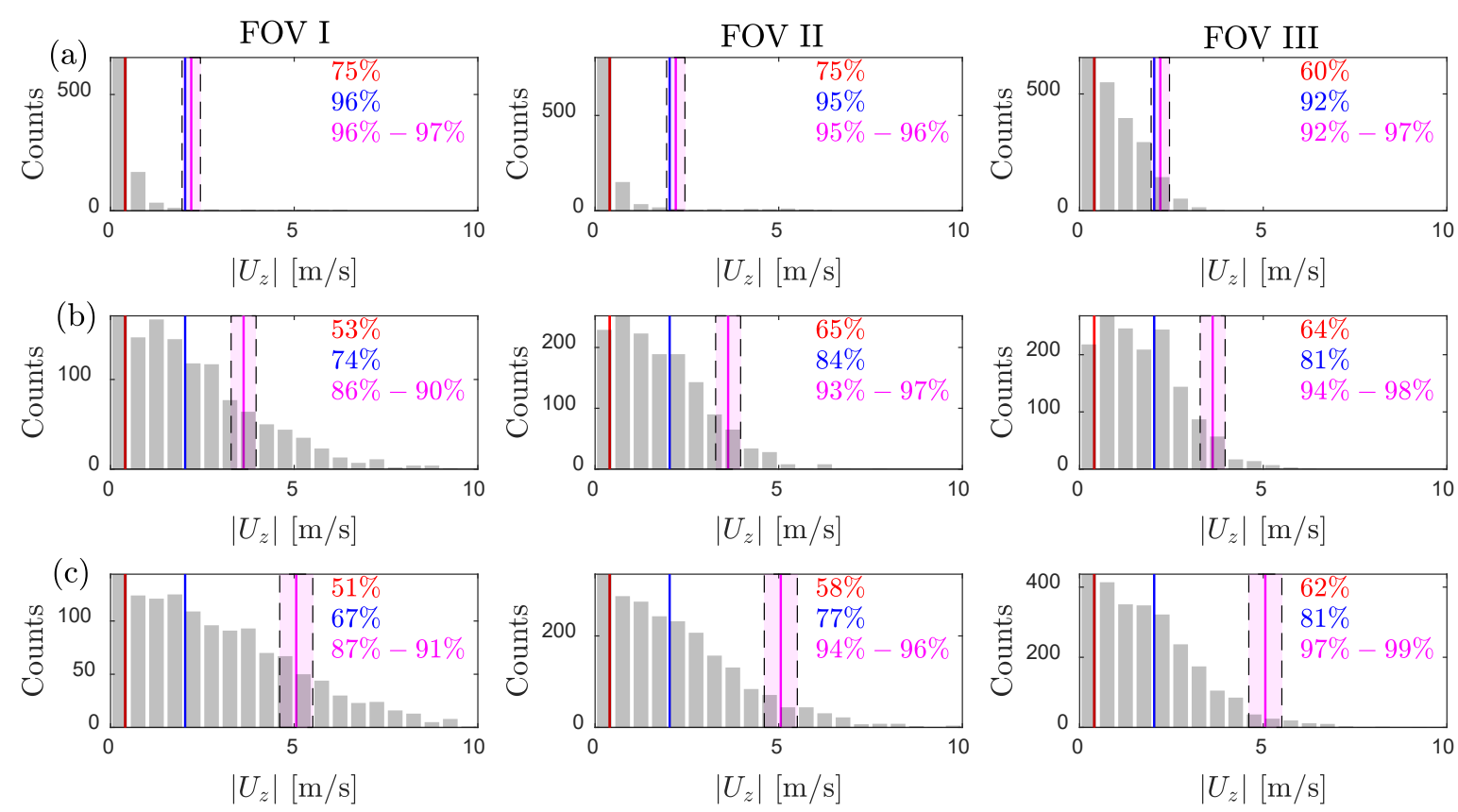

Figure 14: Histograms of the magnitudes of averaged out-of-plane instantaneous velocity within product pocket contours in (a) Dual-A, (b) Dual-C, and (c) Dual-E. Red-vertical lines: $s_{L}$, blue-vertical lines: $s_{L, m a x}$, and magenta-vertical lines: $S_{T} \pm \delta S_{T}$

Results from Figure 14 compare the out-of-plane velocities in the pockets to important flame velocity scales - the laminar flame speed, the flame speed at stretch-induced extinction, and a turbulent flame speed, as was done in our previous work [1]. We assume that pockets that have averaged out-of-plane velocities higher than the turbulent flame speeds may be the result of outof-plane motion and not necessarily a pocket. Using this metric, between $86-99 \%$ of the pockets in a given FOV are actually real pockets, but future analysis will be done to refine this metric. Figure 10 showed that a majority of product gas pockets merge with the main flame front, with some pockets moving out of the FOV and disappearing from the plane of measurement. Unlike reactant pockets, product pockets cannot be consumed. Additionally, merging of these pockets 
with the main flame surface can result in changes in the consumption of reactant gases between these structures and the main flame surface. Whether these structures are pockets or tunnels, it is still important to explore the possibilities due to merging of these flame surfaces.

Assuming the identified structures are actually product pockets, the merging of a product pocket with the reactant side of a flame can influence the local reactant gas consumption either thermally or chemically, as described in recent work by Xu et al. [17]. To understand the potential impact of this internal EGR on local flame propagation, the unstretched laminar flame speed is calculated in Cantera [36] using GRI-Mech 3.0 and results are presented in Figure 15. The goal of this analysis was to understand trends, rather than quantitative effects, of the influence that product gases may have on local flame propagation. In this analysis, three main parameters are varied to quantify the sensitivity of the laminar flame speed of atmospheric methane-air flames at $\phi=1.0: 1$ ) inlet reactant temperatures (sub-figure (a)), 2) addition of cold combustion products as diluents to reactant gases (sub-figure (b)), and 3) addition of hot combustion products at thermodynamic equilibrium temperatures (sub-figures (c)-(d)). In sub-figures (a)-(c), flame speed sensitivity is plotted on the left axis and flame speeds are plotted on the right axis. Results show that an increase in inlet temperature of the reactant gases results in an increase in the laminar flame speeds, whereas the addition of cold combustion products results in a decrease in the laminar flame speed. More realistically, the mixing of product pockets in the reactants results in a diluted but hot reactant gas. The equilibrium temperatures of the reactants mixed with hot combustion products are shown in Figure 15 (d) and the effect of this mixing on the laminar flame speed is shown in Figure 15 (c), understanding that this mixture would likely not have time to equilibrate in the short mixing times ahead of the flame. Trends in sub-figure (c) show that while the addition of diluents decreases the rate at which the flame speed changes, the increase in temperatures has a much greater effect and 
the overall flame speed still increases to up to five times as large as the upstretched laminar flame speed at standard conditions. This result shows that in order to accurately capture local flame propagation velocities, the influence of product pockets must be considered.
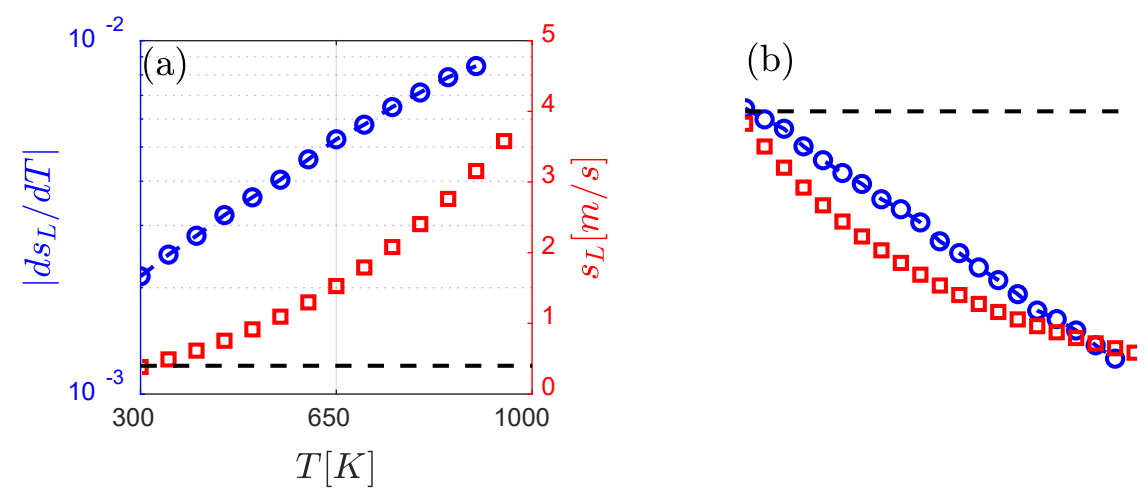

(c)

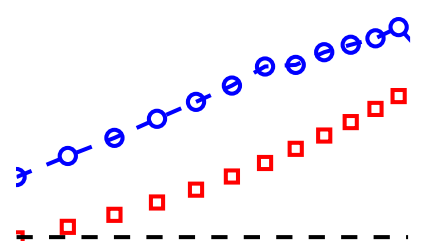

Figure 15: Laminar flame speed variations dues to: (a) preheating of reactants, (b) addition of diluents, and (c) addition of hot combustion products. (d) Equilibrium temperatures as a function of diluent addition. Dashes horizontal lines in sub-figures (a)-(c): $s_{L}=0.4 \mathrm{~m} / \mathrm{s}$

On the other hand, if these product structures are actually flame tunnels, the merging phenomena results in the consumption of reactant gases in the middle of two flame surfaces. DNS studies have shown that this type of interaction can cause flame acceleration in the "pinch" region $[8,37,38]$. This consumption of reactants is estimated by calculating the disappearance of the area between the two structures over a time interval $\Delta t$. The disappearing area is identified using the non-rigid image registration technique, where changes in the flame topology at the location of 
pocket merging are identified and extracted. The rate of this area consumption due to merging $\left(S_{M, C}\right)$ is calculated by using the formulation in Equation (3):

$$
S_{M, C}=\Delta A / P_{1} \Delta t
$$

Where $\Delta A$ is the area consumption due to reactant gas consumption in the plane of measurement for a time interval of $\Delta t$ and $P_{1}$ is the perimeter of the consumed area at any time $t_{0}$. Consumption rates calculated in previous studies also utilize a similar approach to account for the burning of reactant gases $[11,12,14,39]$. Results from these calculations are presented in Figure 16, where PDFs of these consumption rates due to merging events are presented for all flames in FOVs I-III. For reference, the unstretched laminar flame speed is marked with a vertical gray line in each sub-figure. The PDFs shown in this figure have a wide shape with the peaks near a value in the range of $1.5-2.0 \mathrm{~m} / \mathrm{s}$, which is significantly larger than the laminar flame speed at an inlet temperature of $300 \mathrm{~K}$. The analysis described above indicates that merging of these structures, whether they are pockets or tunnels, results in an increase in the burning intensity of reactant gases.
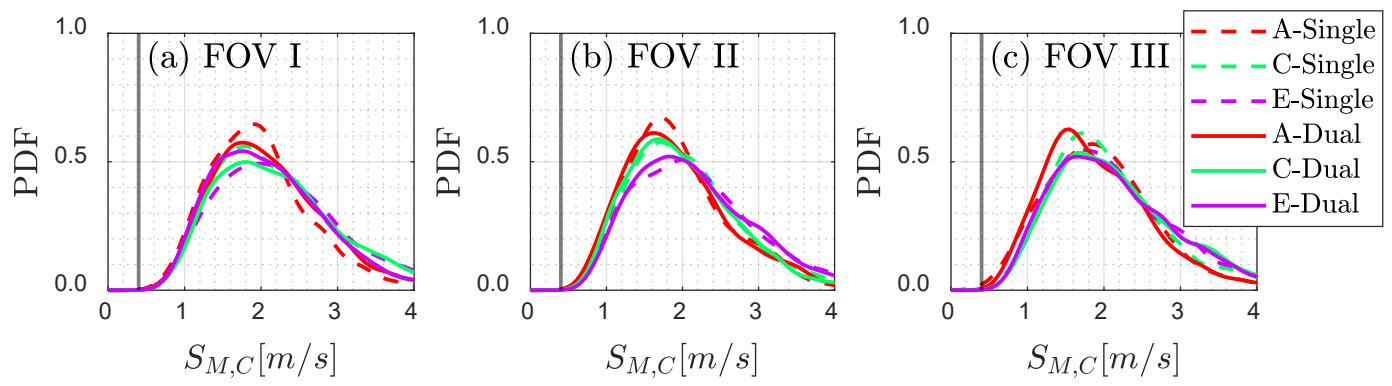

Figure 16: PDFs of reactant gas consumption speeds due to merging of product pockets into flame fronts

\section{Conclusions}

High-speed planar imaging is utilized in the current study to investigate the nature of pocket formation and behavior in turbulent premixed flames in single- and dual-flame configurations. A novel pocket tracking scheme and non-rigid image registration technique is implemented to 
register the lifetime of identified pockets from OH-PLIF images, including their origin and fate. The majority of reactant pockets are formed through flame-flame interactions, whereas the majority of observed product pockets appear from out of plane; this suggests that some of the product pockets we observe may be tunnels reaching through the laser plane. Once formed, reactant and product pockets have very different fates; reactant pockets usually burn out, while product pockets usually merge with the existing flame fronts. These differences in pocket fate result in differences in how the reactants burn, impacting the local burning intensity and heat release rates.

Both the origin and fate of pockets have significant implications for the development of better sub-grid scale models based on flame surface density for performing high-fidelity large-eddy simulations given that most of the pockets are smaller than both the integral and Taylor length scales. Flame-flame interactions that result in pocket formation not only account for local flame surface destruction events on the main flame surface but also generation of freely propagating flame surfaces. While these events behave as a sink and a source term for flame generation, appropriate models may be required to more accurately predict these very different processes. On the other hand, pocket fate also requires special attention. For reactant pockets, global consumption behavior can be modeled as a flame surface sink term, while pocket displacement can be accounted for in the flame surface transport term. Product pockets usually merge with the main flame surface, resulting in addition of flame surface which can be modeled as a flame surface source term. Taking these results into consideration, a better physics-based sub-grid scale model can be generated that can accurately predict the flame behavior and more robustly capture the burning intensity of the reactants.

\section{Acknowledgements}


This work was supported by the Air Force Office of Scientific Research under Grants FA955016-1-0044 and FA9550-16-1-0075 with program manager Dr. Chiping Li. The authors would like to acknowledge Dr. Campbell Carter at the AFRL for providing equipment support for performing these experiments.

\section{References}

[1] A. Tyagi, I. Boxx, S. Peluso, J. O'Connor, Statistics and topology of local flame-flame interactions in turbulent flames, Combustion and Flame 203 (2019) 92-104.

[2] A.W. Skiba, T.M. Wabel, C.D. Carter, S.D. Hammack, J.E. Temme, T. Lee, J.F. Driscoll, Reaction layer visualization: A comparison of two PLIF techniques and advantages of $\mathrm{kHz}-$ imaging, Proceedings of the Combustion Institute 36 (2017) 4593-4601.

[3] J.R. Osborne, S.A. Ramji, C.D. Carter, S. Peltier, S. Hammack, T. Lee, A.M. Steinberg, Simultaneous $10 \mathrm{kHz}$ TPIV, OH PLIF, and $\mathrm{CH} 2 \mathrm{O}$ PLIF measurements of turbulent flame structure and dynamics, Experiments in Fluids 57 (2016) 65.

[4] T. Dunstan, N. Swaminathan, K. Bray, N. Kingsbury, Flame interactions in turbulent premixed twin V-flames, Combustion Science and Technology 185 (2013) 134-159.

[5] T. Dunstan, N. Swaminathan, K. Bray, N. Kingsbury, The effects of non-unity Lewis numbers on turbulent premixed flame interactions in a twin V-flame configuration, Combustion Science and Technology 185 (2013) 874-897.

[6] R. Griffiths, J. Chen, H. Kolla, R. Cant, W. Kollmann, Three-dimensional topology of turbulent premixed flame interaction, Proceedings of the Combustion Institute 35 (2015) 1341-1348. 
[7] B. Denet, Pockets in turbulent premixed flames, Combustion Theory and Modelling 5 (2001) $85-95$.

[8] J.H. Chen, T. Echekki, W. Kollmann, The mechanism of two-dimensional pocket formation in lean premixed methane-air flames with implications to turbulent combustion, Combustion and flame $116(1999)$ 15-48.

[9] C. Sun, C. Law. On the consumption of fuel pockets via inwardly propagating flames. In Symposium (International) on Combustion; 1998: Elsevier. p. 963-970.

[10] R.J. Kee, A Fortran program for modeling steady laminar one-dimensional premixed flames, Sandia Laboratories Report No. SAND 85-8240, (1985).

[11] A.F. Ibarreta, J.F. Driscoll, Measured burning velocities of stretched inwardly propagating premixed flames, Proceedings of the Combustion Institute 28 (2000) 1783-1791.

[12] F. Baillot, A. Bourehla, Burning velocity of pockets from a vibrating flame experiment, Combustion Science and Technology 126 (1997) 201-224.

[13] F. Baillot, D. Durox, D. Demare, Experiments on imploding spherical flames: effects of curvature, Proceedings of the Combustion Institute 29 (2002) 1453-1460.

[14] A. Johchi, Y. Naka, M. Shimura, M. Tanahashi, T. Miyauchi, Investigation on rapid consumption of fine scale unburned mixture islands in turbulent flame via $10 \mathrm{kHz}$ simultaneous CH-OH PLIF and SPIV, Proceedings of the Combustion Institute 35 (2015) 3663-3671. 
[15] D. Han, A. Satija, J.P. Gore, R.P. Lucht, Experimental study of CO 2 diluted, piloted, turbulent CH 4/air premixed flames using high-repetition-rate OH PLIF, Combustion and Flame 193 (2018) $145-156$.

[16] J. Kim, R. Roncancio, A. Satija, R.P. Lucht, J.P. Gore, Correlation between Integral Length Scale and Unburned Pocket Formation in CH4/air Premixed Turbulent Flames, 11th U.S. National Combustion Meeting, Pasadena, California, 2019.

[17] C. Xu, A.Y. Poludnenko, X. Zhao, H. Wang, T. Lu, Structure of strongly turbulent premixed n-dodecane-air flames: Direct numerical simulations and chemical explosive mode analysis, Combustion and Flame 209 (2019) 27-40.

[18] A. Tyagi, I.G. Boxx, S. Peluso, J.A. O'Connor. Statistics of Local Flame-Flame Interactions in Flame Interaction Zones of Two V-Flames. In AIAA Scitech 2019 Forum; 2019. p. 0446.

[19] A. Tyagi, I.G. Boxx, S.J. Peluso, R. Shupp, J. O'Connor. Structure of Flames in Flame Interaction Zones. In 2018 AIAA Aerospace Sciences Meeting; 2018. p. 0161.

[20] N. Peters, Turbulent Combustion, Cambridge University Press (2000).

[21] F. Picano, F. Battista, G. Troiani, C.M. Casciola, Dynamics of PIV seeding particles in turbulent premixed flames, Experiments in Fluids 50 (2011) 75-88.

[22] R.C. Gonzalez, R.E. Woods, Digital image processing prentice hall, Upper Saddle River, NJ, (2002). 
[23] L. Ma, Q. Lei, T. Capil, S.D. Hammack, C.D. Carter, Direct comparison of two-dimensional and three-dimensional laser-induced fluorescence measurements on highly turbulent flames, Opt. Lett. 42 (2017) 267-270.

[24] L. Ma, Q. Lei, J. Ikeda, W. Xu, Y. Wu, C.D. Carter, Single-shot 3D flame diagnostic based on volumetric laser induced fluorescence (VLIF), Proceedings of the Combustion Institute 36 (2017) 4575-4583.

[25] W. Xu, C.D. Carter, S. Hammack, L. Ma, Analysis of 3D combustion measurements using CH-based tomographic VLIF (volumetric laser induced fluorescence), Combustion and Flame 182 (2017) 179-189.

[26] M. Zhang, J. Wang, W. Jin, Z. Huang, H. Kobayashi, L. Ma, Estimation of 3D flame surface density and global fuel consumption rate from 2D PLIF images of turbulent premixed flame, Combustion and Flame 162 (2015) 2087-2097.

[27] X. Li, L. Ma, Capabilities and limitations of 3D flame measurements based on computed tomography of chemiluminescence, Combustion and Flame 162 (2015) 642-651.

[28] S. Trivedi, R. Griffiths, H. Kolla, J. Chen, R.S. Cant, Topology of pocket formation in turbulent premixed flames, Proceedings of the Combustion Institute 37 (2019) 2619-2626.

[29] S. Trivedi, G. Nivarti, R.S. Cant, Flame self-interactions with increasing turbulence intensity, Proceedings of the Combustion Institute 37 (2019) 2443-2449.

[30] J.P. Thirion, Image matching as a diffusion process: an analogy with Maxwell's demons, Medical Image Analysis 2 (1998) 243-260. 
[31] T. Vercauteren, X. Pennec, A. Perchant, N. Ayache, Diffeomorphic demons: Efficient nonparametric image registration, NeuroImage 45 (2009) S61-S72.

[32] N.A. Worth, J.R. Dawson, Characterisation of flame surface annihilation events in self excited interacting flames, Combustion and Flame 199 (2019) 338-351.

[33] S. Sohrab, Z. Ye, C. Law. An experimental investigation on flame interaction and the existence of negative flame speeds. In Symposium (International) on Combustion; 1985: Elsevier. p. $1957-1965$.

[34] P. Trunk, I. Boxx, C. Heeger, W. Meier, B. Böhm, A. Dreizler, Premixed flame propagation in turbulent flow by means of stereoscopic PIV and dual-plane OH-PLIF at sustained $\mathrm{kHz}$ repetition rates, Proceedings of the combustion institute 34 (2013) 3565-3572.

[35] B. Peterson, E. Baum, A. Dreizler, B. Böhm, An experimental study of the detailed flame transport in a SI engine using simultaneous dual-plane OH-LIF and stereoscopic PIV, Combustion and Flame 202 (2019) 16-32.

[36] D.G. Goodwin, H.K. Moffat, R.L. Speth, Cantera: An object-oriented software toolkit for chemical kinetics, thermodynamics, and transport processes. Version 2.2. 1, Cantera Developers, Warrenville, IL, (2016).

[37] T. Echekki, J.H. Chen, I. Gran. The mechanism of mutual annihilation of stoichiometric premixed methane-air flames. In Symposium (International) on Combustion; 1996: Elsevier. p. 855-863.

[38] H.G. Im, J.H. Chen, Preferential diffusion effects on the burning rate of interacting turbulent premixed hydrogen-air flames, Combustion and flame 131 (2002) 246-258. 
[39] M. Shimura, A. Johchi, M. Tanahashi, Consumption rate characteristics of a fine-scale unburnt mixture in a turbulent jet premixed flame by high repetition rate PLIF and SPIV, Journal of Thermal Science and Technology 11 (2016) JTST0047-JTST0047. 